\title{
Gastrointestinal Stromal Tumor as a Rare Cause of Abdominal Mass: A Case Report and Literature Review
}

\author{
Ahmed Baiomi $^{1}$, Hafsa Abbas $^{2}$, Balar Bhavna ${ }^{1}$ \\ 1. Internal Medicine, BronxCare Health System, New York, USA 2. Gastroenterology, BronxCare Health System, New \\ York, USA
}

Corresponding author: Ahmed Baiomi, abaiomi@bronxcare.org

\begin{abstract}
Gastrointestinal stromal tumors (GISTs) are soft tissue sarcomas that can arise from any part of the digestive system. GISTs represent $1 \%$ of gastric neoplasms. We report a rare case of a GIST tumor in a 59-year-old woman who presented with abdominal pain and weight loss. Computed tomography (CT) of the abdomen with intravenous contrast revealed a left upper quadrant abdominal mass and biopsy showed GIST. She was treated with imatinib to downsize the tumor prior to undergoing surgical resection of the tumor.
\end{abstract}

Categories: Gastroenterology, Oncology

Keywords: gastrointestinal stromal tumor (gist), stomach cancer, indeterminate abdominal mass, left sided abdominal pain

\section{Introduction}

GISTs are one of the neoplasms originating from the gastrointestinal tract. They usually present as a subepithelial neoplasm and are often discovered during endoscopy done for various indications. They can originate from any part of the gastrointestinal tract [1]. We present a rare case of GIST where the patient presented with weight loss, abdominal pain and distension.

\section{Case Presentation}

A 59-year-old woman presented to her primary care physician with left-sided abdominal pain for the past eight months associated with a weight loss of 20 pounds. She denied any other GI symptoms. She had no history of smoking, alcohol or drug use. On initial examination in the emergency department, the patient was afebrile, hemodynamically stable and was in no acute distress. Her abdomen was soft, non-tender and there was no organomegaly or mass appreciated. The rest of the review of systems and physical examination was unremarkable. CT scan of abdomen with intravenous contrast revealed a 16 × 16 x $15.4 \mathrm{~cm}$ lesion with a peripheral solid mass and central hypodensity in the left upper quadrant compressing on the spleen, stomach and liver (Figure 1). There was a $3 \mathrm{~mm}$ liver hypodensity. She underwent ultrasound-guided fineneedle aspiration (FNA) biopsy of the lesion and $500 \mathrm{ml}$ of brownish material was aspirated. The

Review began 03/14/2021 Review ended 03/15/2021 Published 03/23/2021

(c) Copyright 2021 Baiomi et al. This is an open access article distributed under the terms of the Creative Commons Attribution License CC-BY 4.0., which permits unrestricted use, distribution, and reproduction in any medium, provided the original author and source are credited. histopathological exam of the specimen showed high-grade gastrointestinal stromal tumor (GIST) of spindle cell type with increased mitotic activity (>10/50 high-power field [HPF]). The immunohistochemical stain revealed the tumor cells were positive for cluster of differentiation (CD)34, CD117, DOG1, and smooth muscle antibodies (SMAs) and negative for CK7, CK20, and S100 antibodies. The KI67 index was approximately $20 \%$ (Figures 2-4). She was evaluated by the oncology and surgery team. She received neoadjuvant chemotherapy with tyrosine kinase inhibitor (imatinib). Follow up CT scan showed good response and the lesion decreased to $10.8 \times 5.0 \times 6.0 \mathrm{~cm}$. She underwent partial gastrectomy with resection of the tumor. The pathology specimen confirmed negative margins. She was advised to continue imatinib for a total of three years post-operatively. Three months after surgery she underwent an upper gastrointestinal endoscopy that showed no residual or recurrent tumor. 


\section{Cureus}

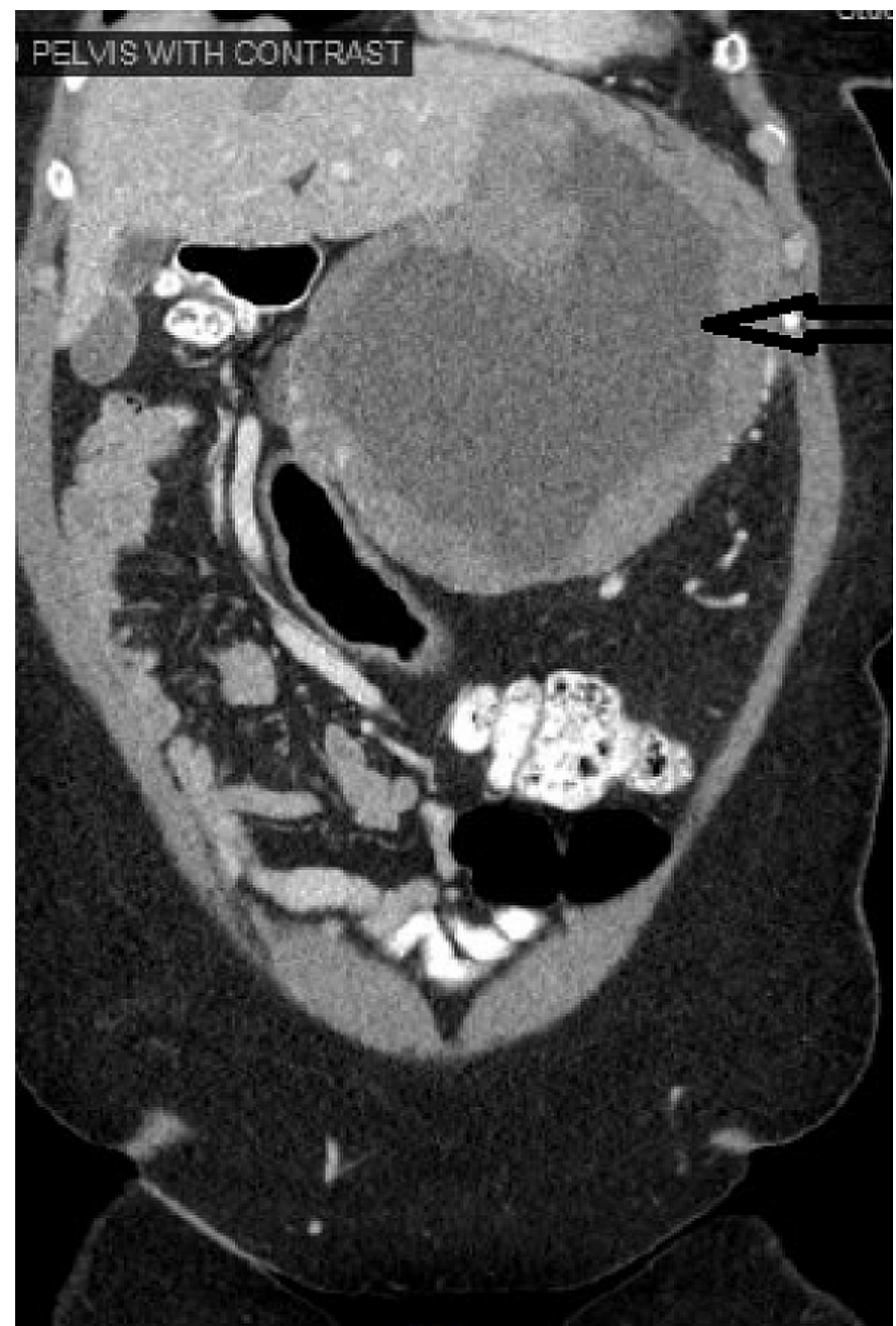

FIGURE 1: Computed Tomography of the abdomen and pelvis with coronal view

Black arrow pointing to the mass 


\section{Cureus}

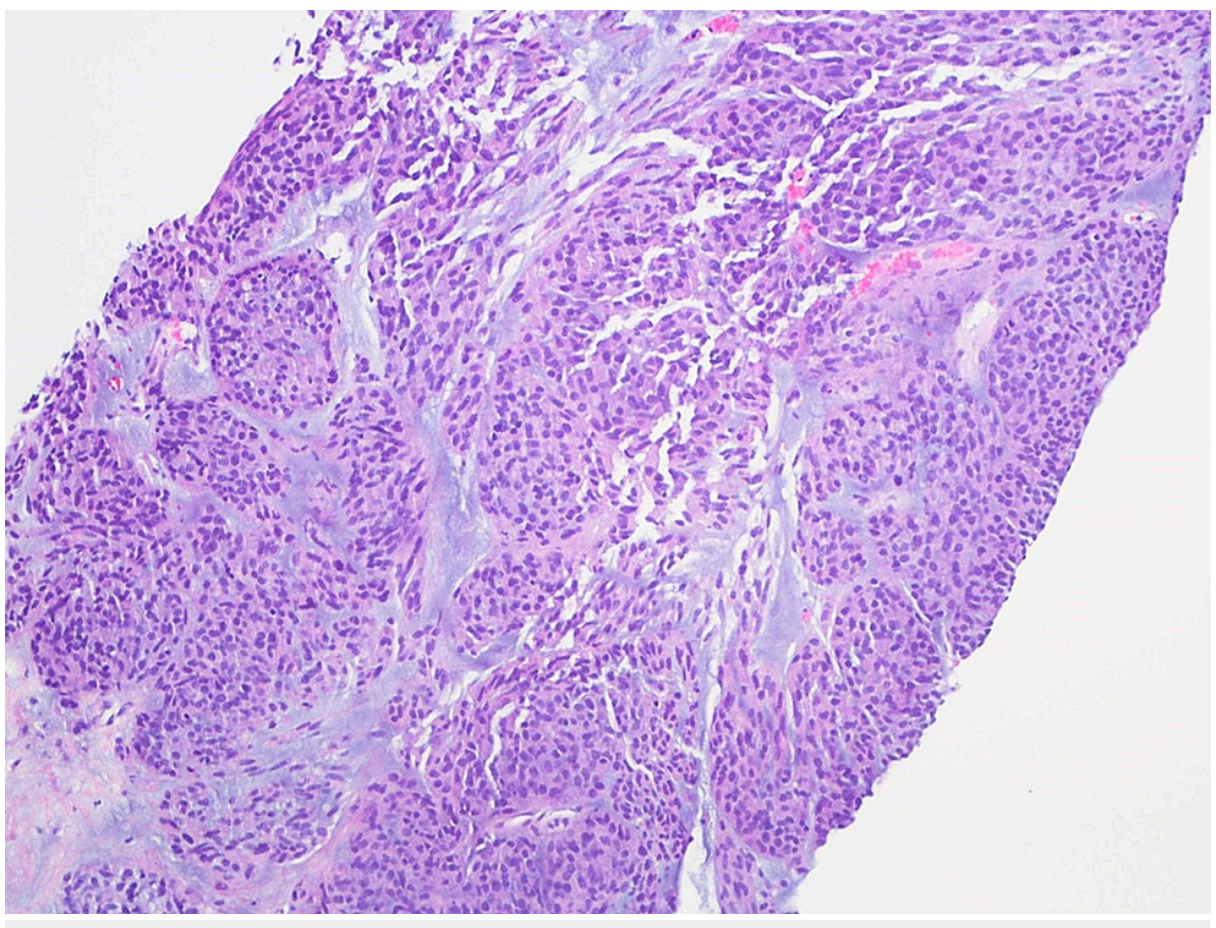

FIGURE 2: Hematoxylin and eosin (H\&E) stain of the mass with magnification $\times 100$

Cellular spindle cell proliferation within loose stroma. The tumor cells are arranged in sheets and nodules.

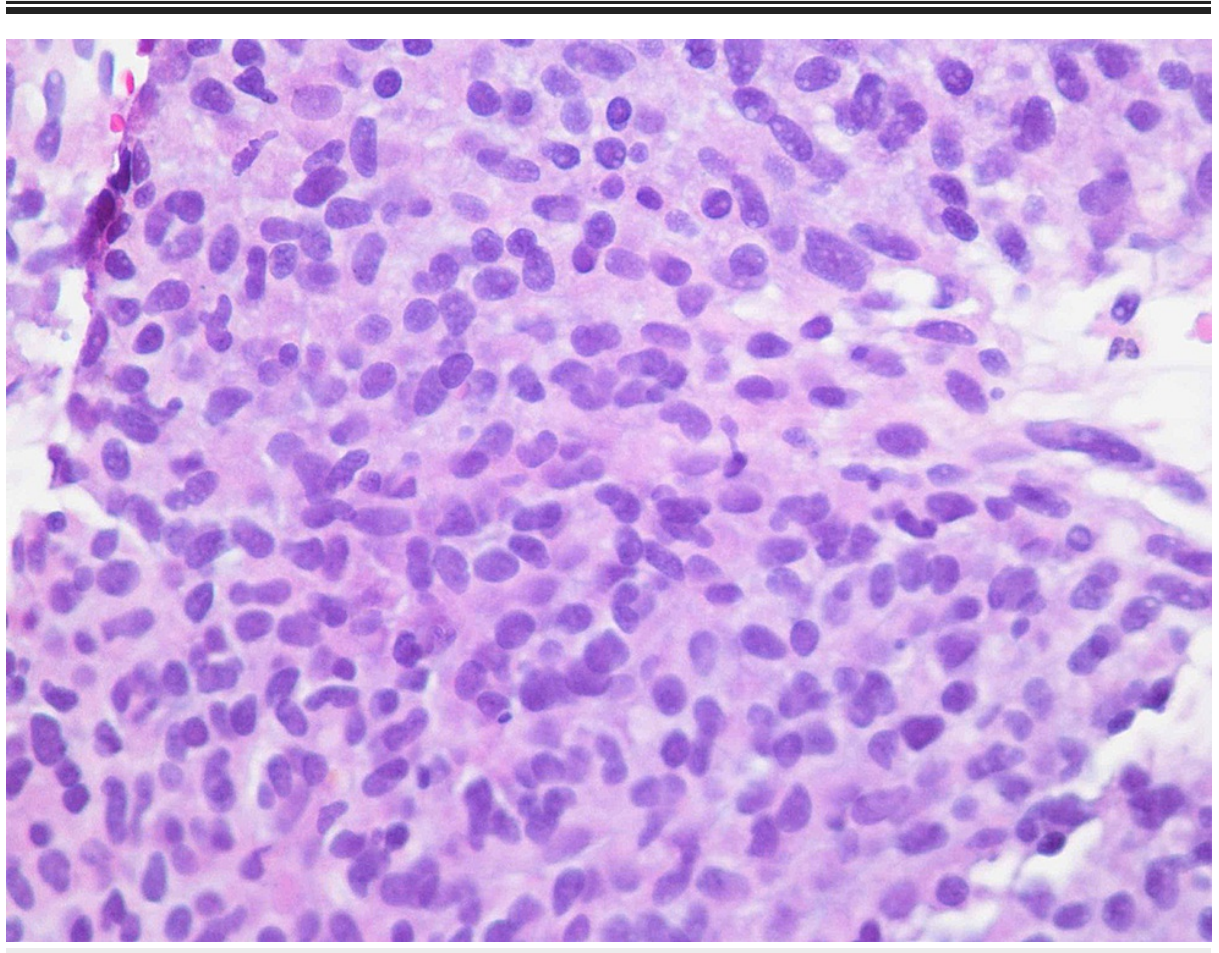

FIGURE 3: Hematoxylin and eosin (H\&E) stain with magnification x 200

Spindle to oval cells with nuclear pleomorphism and arranged in sheets. 


\section{Cureus}

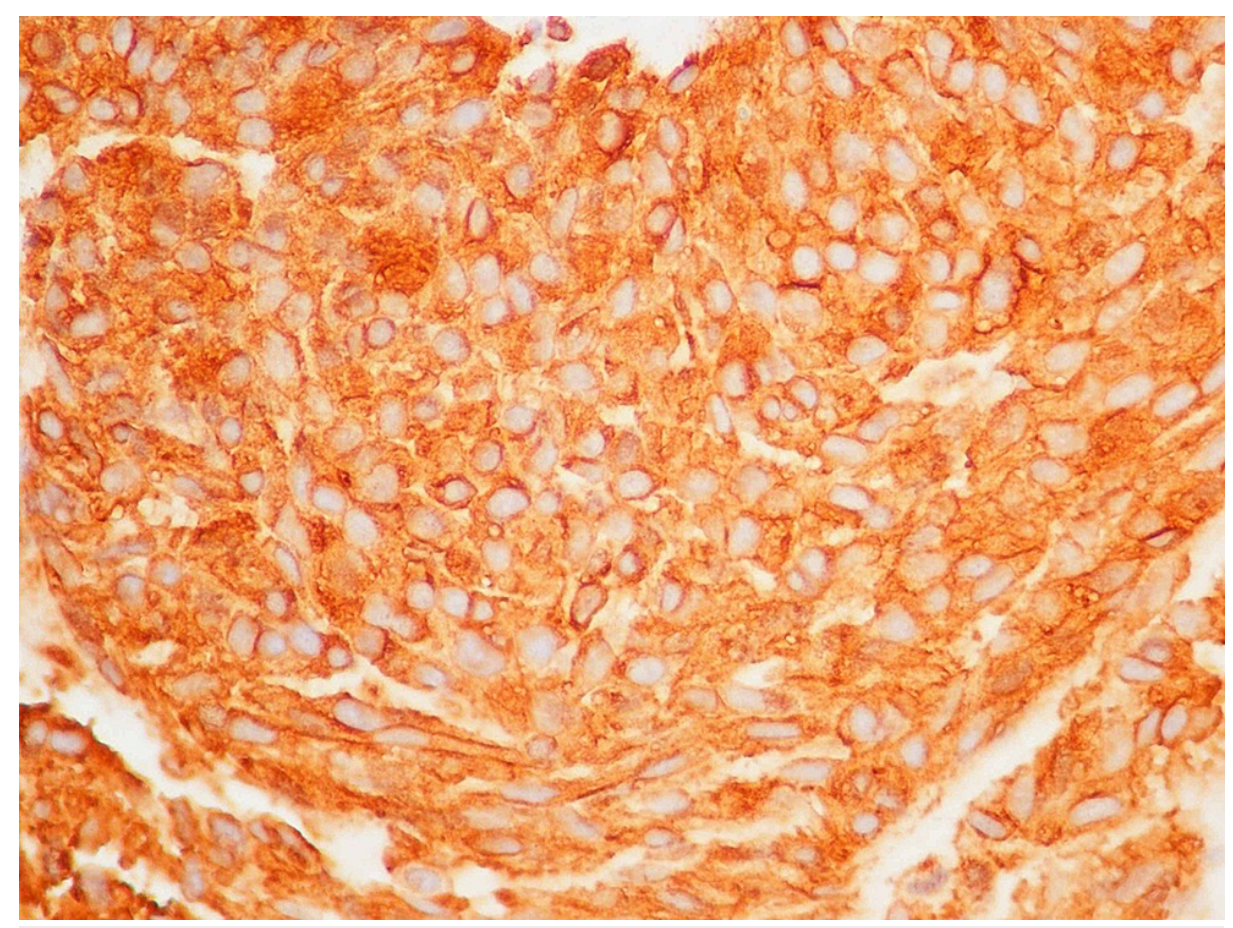

\section{FIGURE 4: Immunohistochemistry, CD117}

The tumor cells are strongly immunoreactive to CD117 (KIT).

\section{Discussion}

GISTs are subepithelial tumors, initially referred to as leiomyoma, leiomyosarcoma or leioblastoma. However, with the availability of electron microscopy and immunohistochemical staining, Mazur and Clarks were able to distinguish GISTs as a separate gastrointestinal neoplasm with distinct features [2].

GISTs represent $1 \%$ of gastrointestinal malignancies with an incidence of seven per million per year [3]. GISTs originate from CD34-positive stem cells residing within the wall of the gut, which can then differentiate incompletely toward the interstitial cells of Cajal (ICC) phenotype. More than $95 \%$ of GISTs exhibit KIT (CD117). Expression of CD34 is not specific for GISTs but is noted to be a poor prognostic indicator as most cases of malignant GIST are CD34 positive. Five percent of GIST cells are not caused through activation and aberrant signaling of the KIT receptor, but rather through mutational activation of the structurally related kinase known as the platelet-derived growth factor receptor- alpha (PDGFRA). Definitive diagnostic criteria for CD117-negative true GIST are currently obscure. The DOG1 gene, which encodes for chloride channel protein actin 1 (independent of KIT and PDGFRA), was discovered in 2004 and is specific for GIST in appropriate clinical and pathological context. Expression of CD117 could be heterogenous, and biopsy could be false negative. True leoisarcomas express smooth muscle actin and desmin but are negative for CD117. Schwannomas are positive for neural antigen S100. GIST tumors are classified into three types based on histopathologic appearance: spindle cell type (70\%), epithelioid type (20\%) or mixed type (10\%) [4-5].

GISTs have a wide variety of clinical presentations depending on the site of involvement. The most common site is the stomach (60-70\%), followed by the small intestine (20-30\%), colon, rectum (5\%), and esophagus $(<5 \%$ [6]. Small GISTs may be asymptomatic and are discovered incidentally during endoscopy. Esophageal GISTs may present with dysphagia [7], and stomach or small intestinal GISTs may present with overt or occult gastrointestinal bleeding [8]. Colonic GISTs may present with acute abdomen, perforation, pain or obstruction [9]. Large tumors may present as an abdominal mass or symptoms of paraneoplastic syndrome. Consumptive hypothyroidism caused by marked overexpression of the thyroid hormone-inactivating enzyme type 3 iodothyronine deiodinase (D3) within GISTs has been reported [10]. Malignant GISTs may present with metastasis most commonly to the liver and peritoneum.

The diagnosis of GIST is often suspected on contrast-enhanced CT or magnetic resonance imaging (MRI) showing an abdominal mass. Imaging can also evaluate the extent of the tumor and assess for the presence of metastasis [11]. Endoscopy can be done to evaluate a luminal involvement by the mass. However, an endoscopic ultrasound (EUS) can differentiate intramural and extramural lesions and can further characterize the mass by identifying its layer of origin and allowing ultrasound-directed fine needle aspiration (FNA) biopsy to be obtained for definitive diagnosis. Usually GISTs are well-demarcated, 
hypoechoic lesions arising from the fourth layer of the gastrointestinal tract (muscularis propria), although small lesions may arise from the second layer (muscular mucosae) [12-13].

GISTs may have different clinical behaviors depending on the site and mitotic activity. In the stomach the benign lesions outnumber the malignant ones, in contrast to the esophagus and colon where most of the GISTs are malignant. Tumors with low mitotic activity, five or fewer mitoses per $50 \mathrm{HPF}$, usually have a benign behavior as compared to those with more than five per 50 HPF are described as malignant. Tumors with more than 50 mitoses per $50 \mathrm{HPF}$ are described as high-grade malignant [14].

Treatment for GIST depends on the tumor size and location. Esophageal GISTs greater than 2 centimeters need to be excised [15], however for those smaller than 2 centimeters different guidelines recommend different management; there is a conservative approach with follow up with repeat esophagogastroduodenoscopy (EGD) and removal if there is an increase in size. Another approach recommends removal of the tumor for fear of the risk of metastasis [16]. For gastric GISTs, submucosal lesions $<1 \mathrm{~cm}$ with EUS findings suggestive of benign tumor may be followed conservatively. Management of gastric lesions between 1 and $2 \mathrm{~cm}$ is controversial and lesions greater than $2 \mathrm{~cm}$ should be excised [17]. For duodenal GISTs, excision is advised whether endoscopically or surgically with pancreaticoduodenectomy [18]. For GISTs in the colon and rectum, excision of the tumor is advised, however sometimes it is challenging especially in the rectum so preoperative imatinib is advised to downsize the tumor and achieve better surgical outcomes [19].

\section{Conclusions}

GISTs comprise only $1 \%$ of gastric neoplasms. Clinical features and management depend on the location, size, appearance and characteristics on EUS. Tumors with benign features can be monitored while large tumors should be excised, with neoadjuvant chemotherapy to downsize the tumor prior to resection. Our patient presented with weight loss and abdominal pain, which is a common symptom of many pathologic conditions. It is important to consider GISTs as one of the differential diagnoses when patients present with similar symptoms, as early stage diagnosis improves outcome and long-term prognosis.

\section{Additional Information}

\section{Disclosures}

Human subjects: Consent was obtained or waived by all participants in this study. Conflicts of interest: In compliance with the ICMJE uniform disclosure form, all authors declare the following: Payment/services info: All authors have declared that no financial support was received from any organization for the submitted work. Financial relationships: All authors have declared that they have no financial relationships at present or within the previous three years with any organizations that might have an interest in the submitted work. Other relationships: All authors have declared that there are no other relationships or activities that could appear to have influenced the submitted work.

\section{References}

1. Rubin BP, Fletcher JA, Fletcher CD: Molecular insights into the histogenesis and pathogenesis of gastrointestinal stromal tumors. Int J Surg Pathol. 2000, 8:5-10. 10.1177/106689690000800105

2. Mazur MT, Clark HB: Gastric stromal tumors. Reappraisal of histogenesis . Am J Surg Pathol. 1983, 7:507519. 10.1097/00000478-198309000-00001

3. Ma GL, Murphy JD, Martinez ME, Sicklick JK: Epidemiology of gastrointestinal stromal tumors in the era of histology codes: results of a population-based study. Cancer Epidemiol Biomarkers Prev. 2015, 24:298-302. 10.1158/1055-9965.epi-14-1002

4. Sakurai S, Fukasawa T, Chong JM, Tanaka A, Fukayama M: C-kit gene abnormalities in gastrointestinal stromal tumors (tumors of interstitial cells of Cajal). Jpn J Cancer Res. 1999, 90:1321-1328. 10.1111/j.13497006.1999.tb00715.x

5. Wang L, Vargas H, French SW: Cellular origin of gastrointestinal stromal tumors: a study of 27 cases . Arch Pathol Lab Med. 2000, 124:1471-1475.

6. Miettinen M, Monihan JM, Sarlomo-Rikala M, Kovatich AJ, Carr NJ, Emory TS, Sobin LH: Gastrointestinal stromal tumors/smooth muscle tumors (GISTs) primary in the omentum and mesentery: clinicopathologic and immunohistochemical study of 26 cases. Am J Surg Pathol. 1999, 23:1109-1118. 10.1097/00000478199909000-00015

7. Miettinen M, Sarlomo-Rikala M, Sobin LH, Lasota J: Esophageal stromal tumors: a clinicopathologic, immunohistochemical, and molecular genetic study of 17 cases and comparison with esophageal leiomyomas and leiomyosarcomas. Am J Surg Pathol. 2000, 24:211-222. 10.1097/00000478-20000200000007

8. Sandrasegaran K, Rajesh A, Rydberg J, Rushing DA, Akisik FM, Henley JD: Gastrointestinal stromal tumors: clinical, radiologic, and pathologic features. AJR Am J Roentgenol. 2005, 184:803-811. 10.2214/air.184.3.01840803

9. Miettinen M, Sarlomo-Rikala M, Sobin LH, Lasota J: Gastrointestinal stromal tumors and leiomyosarcomas in the colon: a clinicopathologic, immunohistochemical, and molecular genetic study of 44 cases. Am J Surg Pathol. 2000, 24:1339-1352. 10.1097/00000478-200010000-00003

10. Maynard MA, Marino-Enriquez A, Fletcher JA, et al.: Thyroid hormone inactivation in gastrointestinal stromal tumors. N Engl J Med. 2014, 370:1327-1334. 10.1056/nejmoa1308893 


\section{Cureus}

11. Scarpa M, Bertin M, Ruffolo C, Polese L, D'Amico DF, Angriman I: A systematic review on the clinical diagnosis of gastrointestinal stromal tumors. J Surg Oncol. 2008, 98:384-392. 10.1002/jso.21120

12. Tio TL, Tytgat GN, den Hartog Jager FC: Endoscopic ultrasonography for the evaluation of smooth muscle tumors in the upper gastrointestinal tract: an experience with 42 cases. Gastrointest Endosc. 1990, 36:342350. 10.1016/s0016-5107(90)71061-9

13. Nagula S, Pourmand K, Aslanian H, et al.: Comparison of endoscopic ultrasound-fine-needle aspiration and endoscopic ultrasound-fine-needle biopsy for solid lesions in a multicenter, randomized trial. Clin Gastroenterol Hepatol. 2018, 16:1307-1313. 10.1016/j.cgh.2017.06.013

14. Franquemont DW: Differentiation and risk assessment of gastrointestinal stromal tumors. Am J Clin Pathol. 1995, 103:41-47. 10.1093/ajcp/103.1.41

15. Lee HJ, Park SI, Kim DK, Kim YH: Surgical resection of esophageal gastrointestinal stromal tumors. Ann Thorac Surg. 2009, 87:1569-1571. 10.1016/j.athoracsur.2009.01.051

16. Blackstein ME, Blay JY, Corless C, et al.: Gastrointestinal stromal tumours: consensus statement on diagnosis and treatment. Can J Gastroenterol. 2006, 20:157-163. 10.1155/2006/434761

17. Sepe PS, Brugge WR: A guide for the diagnosis and management of gastrointestinal stromal cell tumors . Nat Rev Gastroenterol Hepatol. 2009, 6:363-371. 10.1038/nrgastro.2009.43

18. Chok AY, Koh YX, Ow MY, Allen JC, Goh BK: A systematic review and meta-analysis comparing pancreaticoduodenectomy versus limited resection for duodenal gastrointestinal stromal tumors. Ann Surg Oncol. 2014, 21:3429-3438. 10.1245/s10434-014-3788-1

19. Cavnar MJ, Wang L, Balachandran VP, et al.: Rectal gastrointestinal stromal tumor (GIST) in the era of imatinib: organ preservation and improved oncologic outcome. Ann Surg Oncol. 2017, 24:3972-3980. 10.1245/s10434-017-6087-9 